\title{
q-Fermionic Numbers and Their Roles in Some Physical Problems
}

\author{
R.Parthasarathy ${ }^{1}$ \\ The Institute of Mathematical Sciences \\ C.I.T. Campus, Tharamani Post \\ Chennai, 600 113, India.
}

\section{Abstract}

The q-fermion numbers emerging from the q-fermion oscillator algebra are used to reproduce the q-fermionic Stirling and Bell numbers. New recurrence relations for the expansion coefficients in the 'anti-normal ordering' of the qfermion operators are derived. The roles of the q-fermion numbers in qstochastic point processes and the Bargmann space representation for qfermion operators are explored.

\footnotetext{
${ }^{1}$ e-mail address: sarathy@imsc.res.in
} 
q-deformed Stirling numbers were introduced by Carlitz, Gould and Milne [1]. Such numbers are encountered in [2] in the 'normal ordering' of qdeformed boson oscillator creation and annihilation operators [3]. Recently, Schork [4] considered generalized q-Stirling numbers and obtained useful properties. He further introduced unsigned q-deformed Lah numbers. Subsequently, he [5] considered q-fermionic Stirling numbers which are encountered in the 'normal ordering' of q-fermionic oscillator creation and annihilation operators introduced by the author and Viswanathan [6]. Katriel [7] has derived q-Dobinski formula for q-bosonic Bell number by using q-boson coherent states. In contrast to this semi-classical derivation, a probabilistic derivation of the q-Dobinsky formula for q-bosonic Bell number has been obtained by the author and Sridhar [8] by considering q-stochastic point processes.

The Stirling numbers are crucial in many combinatorial problems [9]. Milne [1] in his studies on generalized restricted growth functions obtained qStirling numbers (bosonic), q-Dobinski formula and q-Charlier polynomials. It is very intriguing that these numbers occurring in combinatorial problems, arise naturally in undeformed and q-deformed harmonic oscillator algebra of the creation and annihilation operators.

In this paper we consider q-fermionic numbers in more detail and obtain q-fermionic Stirling and Bell numbers explicitly. Further we consider the 'anti-normal ordering' of the q-deformed bosonic and fermionic operators and obtain new recurrence relations. Using their Fock space states, we give a meaning to these numbers as expressing powers of q-bosonic and q-fermionic numbers in terms of 'raising factorials'. This is the counterpart of the qStirling numbers of the second kind in expressing the powers of q-numbers in terms of 'falling factorials'.

In the subsequent part of this paper, we attempt to give a probabilistic interpretation of the q-fermionic Stirling numbers of the second kind by considering q-stochastic point processes. This introduces q-product densities, a generalization of the concept of product densities introduced in 1950 by Ramakrishnan [10] in his study of cosmic ray cascades. Then, we give a Bargmann space representation of the q-fermion operators as multiplication by and q-differentiation with respect to quasi-Grassmann variable leading to differential equations involving q-differentiation on spaces of entire functions of quasi-Grassmann variable. 


\section{2.q-fermionic numbers}

Macfarlane [3] and Biedenharn [3] introduced q-boson oscillator algebra as

$$
a a^{\dagger}-\sqrt{q} a^{\dagger} a=q^{-N / 2} \quad ; \quad[N, a]=-a \quad, \quad\left[N, a^{\dagger}\right]=a^{\dagger},
$$

where $q>0$. By making a transformation

$$
A=q^{N / 4} a \quad ; \quad A^{\dagger}=a^{\dagger} q^{N / 4}
$$

one obtains

$$
A A^{\dagger}-q A^{\dagger} A=1 ; q>0
$$

and the associated q-bosonic number

$$
[n]_{b}=\frac{1-q^{n}}{1-q}
$$

In the above $q$ is strictly positive. The author and Viswanathan [6], proposed a non-trivial q-fermion oscillator algebra as

$$
f f^{\dagger}+\sqrt{q} f^{\dagger} f=q^{-N / 2} ;[N, f]=-f,\left[N, f^{\dagger}\right]=f^{\dagger}, f^{2} \neq 0,\left(f^{\dagger}\right)^{2} \neq 0(5)
$$

where $q>0$. By making a transformation

$$
F=q^{N / 4} f \quad ; \quad F^{\dagger}=f^{\dagger} q^{N / 4}
$$

one obtains

$$
F F^{\dagger}+q F^{\dagger} F=1 \quad ; \quad F^{2} \neq 0,\left(F^{\dagger}\right)^{2} \neq 0, q>0
$$

and the associated q-fermionic number

$$
[n]_{f}=\frac{1-(-1)^{n} q^{n}}{1+q}
$$

In (5) to (8), $q$ is strictly positive. Thus, it is not correct to replace $q$ by -q in the q-boson algebra to obtain q-fermion algebra (7), as q in (1) to (4) 
is strictly positive. Nevertheless, for mathematical expressions such replacement may be carried out. In what follows, we shall denote (8) by q-fermion number. This definition is of fundamental importance and is quite different from $[n]_{b}$. In the limit $q \rightarrow 1,[n]_{b} \rightarrow n$ while $[n]_{f} \rightarrow \frac{1}{2}\left(1-(-1)^{n}\right)$ taking values 0 and 1 for $n$ even and odd. The properties of the q-fermion numbers associated with (5) (which are different from (8)) have been studied by Narayana Swamy [11]. For $q<1$, the q-fermion numbers (8) never go beyond 1 for any value of $n$ and for $n \rightarrow \infty$, it asymptotically approches 0.5 . On the other hand the q-boson numbers (4), become the usual numbers when $q=1$, and for $q<1$, as $n \rightarrow \infty$ we have, $[n]_{b}$ asymptotically goes to $1 /(1-q)$. For $q>1$, we have for q-fermion numbers, $[0]_{f}=0,[1]_{f}=1$ and $[n]_{f}>0$ for $n$ odd, $<0$ for $n$ even.

The q-fermions described by (5) or (7) with the q-numbers (8) are different from the k-fermions introduced by Daoud, Hassouni and Kibler [12]. The kfermion algebra is a non-Hermitian realization of the q-deformed Heisenberg bosonic algebra with $q$ being a root of unity and satisfy $f_{ \pm}^{k}=0$ and $f_{+} \neq f_{-}^{\dagger}$, except for $k=2$, for which they become ordinary fermions. On the other hand, q-fermions admit $q$ real or complex and only when $q=1$, they become ordinary fermions.

\section{Normal Ordering of q-fermion operators}

We wish to evaluate $\left(F^{\dagger} F\right)^{r}$ using (7). It is straightforward to expand,

$$
\left(F^{\dagger} F\right)^{r}=\sum_{s=1}^{r} \mathcal{F}_{s}^{r}\left(F^{\dagger}\right)^{s} F^{s},
$$

and find a recurrence relation for $\mathcal{F}_{s}^{r}$. From (9), it follows

$$
\left(F^{\dagger} F\right)^{r+1}=\sum_{s=1}^{r} \mathcal{F}_{s}^{r}\left(F^{\dagger}\right)^{s} F^{s} F^{\dagger} F .
$$

From (7), we have

$$
F^{s} F^{\dagger}=[s]_{f} F^{s-1}+(-1)^{s} q^{s} F^{\dagger} F^{s} .
$$

Using (11) in (10) and noting from $(9), \mathcal{F}_{0}^{r}=0, \mathcal{F}_{r+1}^{r}=0$, we find

$$
\mathcal{F}_{s}^{r+1}=(-1)^{s-1} q^{s-1} \mathcal{F}_{s-1}^{r}+[s]_{f} \mathcal{F}_{s}^{r},
$$


the desired recurrence relation for $\mathcal{F}_{s}^{r}$ coefficients in (9). The recurrence relation (12) with $\mathcal{F}_{1}^{1}=1$ is the same as that of the q-fermionic Stirling numbers of the second kind [5].

The q-fermionic Bell number introduced in [5] is

$$
\mathcal{B}_{r}^{(f)}=\sum_{s=1}^{r} \mathcal{F}_{s}^{r}
$$

An attempt along the lines of [7] for q-fermionic Bell number runs into difficulty. q-fermion coherent states have been constructed in [13] using 'quasi Grassmann' variables $\psi$. It is to be noted that the replacement of $q$ by $-q$ in the q-boson coherent states will not give q-fermion coherent states. These two coherent states are structurally very different. As $\psi^{\dagger} \psi+\psi \psi^{\dagger}=0 ; \psi^{2} \neq$ $0,\left(\psi^{\dagger}\right)^{2} \neq 0$, it is not possible to use the analogue of $|z|=1$ here. So, we take the matrix elements of (9) between q-fermion Fock space states [13] $\mid n>$ with $n>r$ and use $F\left|n>=\sqrt{[n]_{f}}\right| n-1>; F^{\dagger}\left|n>=\sqrt{[n+1]_{f}}\right| n+1>$ to arrive at

$$
[n]_{f}^{r}=\sum_{s=1}^{r} \mathcal{F}_{s}^{r} \frac{[n]_{f} !}{[n-s]_{f} !}
$$

which can be verified explicitly using (8) and (12). Multiplying (14) by $\lambda^{n}$ and summing $n$ from 1 to $\infty$ and then setting $\lambda=1$, we obtain

$$
\mathcal{B}_{r}^{(f)}=\left(e_{q}^{(f)}(1)\right)^{-1} \sum_{n=1}^{\infty} \frac{[n]_{f}^{r}}{[n]_{f} !},
$$

where

$$
e_{q}^{(f)}(x)=\sum_{n=0}^{\infty} \frac{x^{n}}{[n]_{f} !} .
$$

(15) is the q-fermionic Dobinski formula.

Some of the q-fermionic Bell numbers are:

$$
\begin{aligned}
& \mathcal{B}_{1}^{(f)}=1, \\
& \mathcal{B}_{2}^{(f)}=1-q,
\end{aligned}
$$




$$
\begin{aligned}
\mathcal{B}_{3}^{(f)} & =1-q-q[2]_{f}-q^{3} \\
\mathcal{B}_{4}^{(f)} & =1-q-q[2]_{f}-q[2]_{f}^{2}-q^{3}-q^{3}[2]_{f}-q^{3}[3]_{f}+q^{6} \\
\mathcal{B}_{5}^{(f)} & =1-q+\left(-q-q[2]_{f}-q[2]_{f}^{2}\right)\left([2]_{f}+q^{2}\right) \\
& +\left(-q^{3}-q^{3}[2]_{f}-q^{3}[3]_{f}\right)\left([3]_{f}-q^{3}\right)+q^{6}[4]_{f}+q^{10} .
\end{aligned}
$$

In the limit $q=1$, we have

$$
\begin{aligned}
& \mathcal{B}_{1}^{(f)}=1 \quad ; \quad \mathcal{B}_{2}^{(f)}=0, \\
& \mathcal{B}_{r}^{(f)}=(-1)^{r} \quad \text { if } r=0(\bmod 3) \\
& (-1)^{r+1} \quad \text { if } r=1(\bmod 3) \\
& 0 \quad \text { if } r=2(\bmod 3) \text {. }
\end{aligned}
$$

The results (17) have been obtained by Wagner [14] in his study of generating functions for some well known statistics on the family of partitions of a finite set.

The q-fermionic Stirling numbers of the first kind are introduced by expressing the inverse of $(9)$, as

$$
\left(F^{\dagger}\right)^{r} F^{r}=\sum_{s=1}^{r} \mathcal{S}_{s}^{r}\left(F^{\dagger} F\right)^{s}
$$

The matrix elements of the above between q-fermion Fock space states $|n\rangle$ ,$(n>r)$ give

$$
\frac{[n]_{f} !}{[n-r]_{f} !}=\sum_{s=1}^{r} \mathcal{S}_{s}^{r}[n]_{f}^{s} .
$$

Using $[n]_{f}-[r]_{f}=(-1)^{r} q^{r}[n-r]_{f}$, we have the recurrence relation

$$
\mathcal{S}_{s}^{r+1}=(-1)^{r} q^{-r} \mathcal{S}_{s-1}^{r}-[r]_{f}(-1)^{r} q^{-r} \mathcal{S}_{s}^{r} .
$$

Similarly, the q-fermionic unsigned Lah numbers introduced as

$$
\frac{[r+n-1]_{f} !}{[r-1]_{f} !}=\sum_{s=0}^{n} \mathcal{L}_{s}^{n} \frac{[r]_{f} !}{[n-s]_{f} !}
$$


have the recurrence relation

$$
\mathcal{L}_{s}^{n+1}=(-1)^{n+s-1} q^{n+s-1} \mathcal{L}_{s-1}^{n}+[s+n]_{f} \mathcal{L}_{s}^{n}
$$

These mathematical results reveal the feature of obtaining them from their q-bosonic counterparts by replacing $q$ by $-q$. The unsigned q-Lah numbers are met in the normal ordering of $\left(\left(A^{\dagger}\right)^{r} A^{s}\right)^{n}$ for $r=2$ and $s=1$ [15] and their q-analogues have been obtained in $[4,5]$.

\section{Anti-Normal Ordering of q-fermion operators}

In this section, we seek an expansion for $\left(A A^{\dagger}\right)^{r}$. First, we consider qbosonic operators in (3). It is straightforward to expand

$$
\left(A A^{\dagger}\right)^{r}=\sum_{s=1}^{r} \mathcal{A}_{s}^{r} A^{s}\left(A^{\dagger}\right)^{s},
$$

and use

$$
\left(A^{\dagger}\right)^{s} A=\frac{1}{q^{s}} A\left(A^{\dagger}\right)^{s}-\frac{1}{q^{s}}[s]\left(A^{\dagger}\right)^{s-1}
$$

to obtain a recurrence relation for $\mathcal{A}_{s}^{r}$ with $\mathcal{A}_{1}^{1}=1, \mathcal{A}_{0}^{r}=0, \mathcal{A}_{r+1}^{r}=0$ as

$$
\mathcal{A}_{s}^{r+1}=q^{-(s-1)} \mathcal{A}_{s-1}^{r}-[s] q^{-s} \mathcal{A}_{s}^{r} .
$$

A similar relation for q-fermionic operators anti-normal orderering, namely

$$
\left(F F^{\dagger}\right)^{r}=\sum_{s=1}^{r} \mathcal{B}_{s}^{r} F^{s}\left(F^{\dagger}\right)^{s},
$$

can be obtained from (7). We have from (7)

$$
\left(F^{\dagger}\right)^{s} F=(-1)^{s} q^{-s} F\left(F^{\dagger}\right)^{s}-(-1)^{s} q^{-s}[s]_{f}\left(F^{\dagger}\right)^{s-1} .
$$

Using this and (24), we find $\left(\mathcal{B}_{1}^{1}=1 ; \mathcal{B}_{0}^{r}=0 ; \mathcal{B}_{r+1}^{r}=0\right)$,

$$
\mathcal{B}_{s}^{r+1}=(-1)^{s-1} q^{-(s-1)} \mathcal{B}_{s-1}^{r}-(-1)^{s} q^{-s}[s]_{f} \mathcal{B}_{s}^{r} .
$$


These recurrence relations (23) and (25) are different from those of q-Stirling or Lah numbers.

In order to obtain a relationship between $\mathcal{A}_{s}^{r}\left(\mathcal{B}_{s}^{r}\right)$ and $[n]_{b}\left([n]_{f}\right)$, we use the q-boson Fock space states for (22) and q-fermion Fock space for (24). Then using the standard action of the creation and annihilation q-operators on the Fock space states, we find

$$
\begin{aligned}
{[n+1]_{b}^{r} } & =\sum_{s=1}^{r} \mathcal{A}_{s}^{r} \frac{[n+s]_{b} !}{[n]_{b} !} \\
{[n+1]_{f}^{r} } & =\sum_{s=1}^{r} \mathcal{B}_{s}^{r} \frac{[n+s]_{f} !}{[n]_{f} !}
\end{aligned}
$$

It is interesting to observe that while the normal ordering of operators yielded expressions for $[n]_{b}^{r}\left([n]_{f}^{r}\right)$ in terms of q-stirling numbers of the second kind as 'falling factorials' (namely (14) and its q-bosonic analogue), the anti-normal ordering yields expressions for $[n]_{b}^{r}\left([n]_{f}^{r}\right)$ in terms of $\mathcal{A}_{s}^{r}, \mathcal{B}_{s}^{r}$ as 'raising factorials'.

\section{5.q-fermionic Stirling number of second kind - a probabilistic view}

In this section we extend the theory of product densities of Ramakrishnan $[10]$ to a q-extension of the stochastic variable $[n(E)]_{f}$ which depend on a continuous parameter $E$ taken to be ordinary variable, The statistical properties of these q-fermionic stochastic variable taking values $[n(E)]_{f}$ will be governed by q-stochastic point processes. Recall that $[n]_{f}$ for $q<1$ never exceeds unity. So the feature assumed in [10] namely atmost one particle occurs in the interval $d E$ is maintained. Now we define the q-number of particles in the range $E$ and $E+d E$ to be $[n(E+d E)-n(E)]_{f}$ which is just $[d n(E)]_{f}$. Following [10], we take the probability that there occurs $[1]_{f}=1$ particle in the interval $d E$ is proportional to $d E$ and that for the occurrence of $[n]_{f}$ particles in $d E$ is proportional to $(d E)^{n}$. The average number of particles in the interval $d E$, denoted by $\mathcal{E}\left([d n(E)]_{f}\right)$, is represented by a q-function $f_{1}^{(q)}(E)$ such that

$$
\mathcal{E}\left([\operatorname{dn}(E)]_{f}\right)=f_{1}^{(q)}(E) d E .
$$


Denoting the probability that $[n]_{f}$ particles occuring in the interval $d E$ by $P_{q}\left([n]_{f}\right)$, we have

$$
\begin{aligned}
P_{q}\left([1]_{f}\right) & \equiv P_{q}(1)=f_{1}^{(q)}(E) d E+\mathcal{O}\left((d E)^{2}\right) \\
P_{q}\left([0]_{f}\right) & \equiv P_{q}(0)=1-f_{1}^{(q)}(E) d E-\mathcal{O}\left((d E)^{2}\right) \\
P_{q}\left([n]_{f}\right) & =\mathcal{O}\left((d E)^{n}\right) ; n>1
\end{aligned}
$$

The average of the $r^{t h}$ moment of $[n]_{f}$ is then

$$
\begin{aligned}
\mathcal{E}\left([n]_{f}^{r}\right) & =\sum_{n}[n]_{f}^{r} P_{q}([n]) \\
& =f_{1}^{(q)}(E) d E=\mathcal{E}([d n(E)])
\end{aligned}
$$

where the second step follows from (27) and (28). Thus all the moments are equal to the probability that the q-stochastic variable assumes the value $[1]_{f}=1$. This feature of $[10]$ is maintained here. $f_{1}^{(q)}(E)$ is the q-product density of degree 1 .

Now we consider the distribution of $[n]_{f}$ particles in the $E$-axis, that is, in the intervals $d E_{1}, d E_{2}, \cdots d E_{n}$, with $[1]_{f}(=1)$ particle in each interval. For the first interval $d E_{1}$, this can be done in ${ }^{[n]_{f}} C_{[1]_{f}}=[n]_{f} ! /[n-1]_{f} !=[n]_{f}$ number of ways, where ${ }^{[n]_{f}} C_{[1]_{f}}$ is the q-binomial coefficient. Thus, from the average number of particles in (27), we have

$$
f_{1}^{(q)}\left(E_{1}\right) d E_{1}=[n]_{f} f_{1}^{(q) 0}\left(E_{1}\right) d E_{1},
$$

with

$$
\int_{\text {whole range }} f_{1}^{(q) 0}(E) d E=1 .
$$

If we now use the reamaining particles as $\left([n]_{f}-1\right)$, then the number of ways of putting $[1]_{f}$ particle in $d E_{2}$ will be $[n]_{f}-1$. Then the joint probability of putting $[1]_{f}$ particle each in $d E_{1}$ and $d E_{2}$ will be proportional to $[n]_{f}\left([n]_{f}-1\right)$, if we were to use the same product density in [10]. But, in this way, we will not be exhausting the total number $[n]_{f}$ of particles. So, in dealing with qnumbers, it is necessary to introduce q-product densities, such that the joint 
probability of putting $[1]_{f}$ particle each in $d E_{1}$ and $d E_{2}$ will be taken to be proportional to $[n]_{f}[n-1]_{f}$. Then the average number in this case will be

$$
\begin{aligned}
\mathcal{E}\left(\left[d n\left(E_{1}\right)\right]_{f}\left[d n\left(E_{2}\right)\right]_{f}\right) & \equiv f_{2}^{(q)}\left(E_{1}, E_{2}\right) d E_{1} d E_{2} \\
& =[n]_{f}[n-1]_{f} f_{1}^{(q) 0}\left(E_{1}\right) f_{1}^{(q) 0}\left(E_{2}\right) d E_{1} d E_{2}
\end{aligned}
$$

Proceeding further, the joint probability of putting $[1]_{f}$ particle each in $d E_{1}, d E_{2}, \cdots d E_{n}$ will be proprtional to $[n]_{f} !$, thereby exhausting the total number $[n]_{f}$ particles. This gives

$$
\begin{aligned}
f_{m}^{(q)}\left(E_{1}, \cdots E_{m}\right) d E_{1} \cdots d E_{m} & =\frac{[n]_{f} !}{[n-m]_{f} !} f_{1}^{(q) 0}\left(E_{1}\right) \cdots f_{1}^{(q) 0}\left(E_{m}\right) d E_{1} \cdots d E_{m} \\
f_{n}^{(q)}\left(E_{1} \cdots E_{n}\right) d E_{1} \cdots d E_{n} & =[n]_{f} ! f_{1}^{(q) 0}\left(E_{1}\right) \cdots f_{1}^{(q) 0}\left(E_{n}\right) d E_{1} \cdots d E_{n} .
\end{aligned}
$$

In (32) and (33), the intervals do not overlap. When the intervals overlap, a degeneracy occurs [10] and then for a finite interval $\triangle E=E_{u}-E_{t}$

$$
\begin{aligned}
\int_{E_{t}}^{E_{u}} \int_{E_{t}}^{E_{u}} \mathcal{E}\left(\left[\operatorname{dn}\left(E_{1}\right)\right]_{f}\left[\operatorname{dn}\left(E_{2}\right)\right]_{f}\right) & =\int_{E_{t}}^{E_{u}} f_{1}^{(q)}(E) d E \\
& +\int_{E_{t}}^{E_{u}} \int_{E_{t}}^{E_{u}} f_{2}^{(q)}\left(E_{1}, E_{2}\right) d E_{1} d E_{2} .(34
\end{aligned}
$$

The $r^{\text {th }}$ moment of the q-number of particles in the finite range $\triangle E$, namely $\mathcal{E}\left([n]_{f}^{r} \triangle E\right)$ can be represented, after taking the degeneracy into account, by

$$
\mathcal{E}\left([n]_{f \triangle E}^{r}\right)=\sum_{s=1}^{r} \mathcal{C}_{s}^{r} \int_{E_{t}}^{E_{u}} \cdots \int_{E_{t}}^{E_{u}} f_{s}^{(q)}\left(E_{1}, \cdots E_{s}\right) d E_{1} \cdots d E_{s},
$$

where the coefficients $\mathcal{C}_{s}^{r}$ are functions of $r$ and $s$ alone. For $[n]_{f}$ fixed, integrating over the whole range and using (31) and (33), we obtain

$$
[n]_{f}^{r}=\sum_{s=1}^{r} \mathcal{C}_{s}^{r} \frac{[n]_{f} !}{[n-s]_{f} !}
$$

which is same as (14) upon identifying $\mathcal{C}_{s}^{r}$ with $\mathcal{F}_{s}^{r}$. This derivation of (36) gives the role of the q-fermionic Strirling number of the second kind as taking into account the degenarcaies in the joint probabilities of the distribution of q-fermionic number $[n]_{f}$ as a stochastic variable when the intervals overlap. 


\section{Bargmann Space Representation for q-fermion operators}

A Bargmann space realization for q-bosons has been developed by Bracken, MacAnally, Zhang and Gould [16] and that for q-fermions has been developed by the author [17]. As the q-fermion coherent states involve quasi-Grassmann variable $\psi$, the space consists of monomials of $\psi$. In this section, we briefly recall the main results to illustrate two points. First, a naive replacement of $q$ by $-q$ is not sufficient and is incorrect. Second, we would like to represent (9) and (24) in terms of 'differential operators'. The q-fermion coherent state [13] is given by

$$
\begin{aligned}
\mid \psi> & =\left(e_{q}^{\psi^{\dagger} \psi}\right)^{-\frac{1}{2}} e_{q}^{-\psi F^{\dagger}} \mid 0>, \\
& =\left(e_{q}^{\psi^{\dagger} \psi}\right)^{-\frac{1}{2}} \sum_{n=0}^{\infty}(-1)^{n}\left\{\frac{\psi^{2 n}}{\sqrt{[2 n]_{f} !}} \mid 2 n>\right. \\
& \left.-\frac{\psi^{2 n+1}}{\sqrt{[2 n+1]_{f} !}} \mid 2 n+1>\right\},
\end{aligned}
$$

and it can be verified $F|\psi>=\psi| \psi>$. We will first map a vector in the Hilbert space $\mid \phi>$ to a function $\phi(\psi)$ by

$$
\begin{aligned}
\phi(\psi) & \equiv<\psi^{\dagger} \mid \phi>, \\
& =\left(e_{q}^{\psi^{\dagger} \psi}\right)^{-\frac{1}{2}} \sum_{n=0}^{\infty}(-1)^{n}\left\{\frac{\psi^{2 n}}{\sqrt{[2 n]_{f} !}}<2 n \mid \phi>\right. \\
& \left.+\frac{\psi^{2 n+1}}{\sqrt{[2 n+1]_{f} !}}<2 n+1 \mid \phi>\right\},
\end{aligned}
$$

where we have made use of the anti-commuting property of $\psi$ with $F$ and $F^{\dagger}$. In [17] it has been shown that $\phi(\psi)$ is an entire function.

Now consider the matrix element $\left\langle\psi^{\dagger}\left|F^{\dagger}\right| \phi\right\rangle$. Using $F|\psi\rangle=\psi \mid \psi>$, we have

$$
<\psi^{\dagger}\left|F^{\dagger}\right| \phi>=\psi \phi(\psi)
$$

and so in the space of $\phi(\psi), F^{\dagger}$ is represented by multiplication by $\psi$. Consider now the expression $\psi<\psi^{\dagger}|F| \phi>$ which can rewritten as

$$
\psi<\psi^{\dagger}|F| \phi>=<\psi^{\dagger}\left|F^{\dagger} F\right| \phi>\text {. }
$$


Writing $F^{\dagger} F$ as $[N]_{f}$ (which is possible in view of the expression for $\mid \psi>$ in terms of expansion of q-fermion Fock space states), we have

$$
\begin{aligned}
\psi<\psi^{\dagger}|F| \phi> & =<\psi^{\dagger}\left|[N]_{f}\right| \phi>, \\
& =<\psi^{\dagger}\left|\frac{1-(-1)^{N} q^{N}}{1+q}\right| \phi>,
\end{aligned}
$$

where in the last step we used (8). Using (38), we have $\left\langle\psi^{\dagger}\left|q^{N}\right| \phi\right\rangle=\phi(q \psi)$ with the prefactor in (38) unaltered and so,

$$
\psi<\psi^{\dagger}|F| \phi>=\frac{\phi(\psi)-\phi(-q \psi)}{1+q} .
$$

This suggests to introduce q-differentiation as

$$
\frac{d_{q}}{d_{q} \psi} \phi(\psi) \equiv \frac{\phi(\psi)-\phi(-q \psi)}{\psi(1+q)} .
$$

Thus in the space of $\phi(\psi), F$ is represented by q-differentiation with respect to $\psi$. It can be verified $\frac{d_{q}}{d_{q} \psi} \psi^{n}=[n]_{f} \psi^{n-1}$. In this way, we realize that the Bargmann spaces for q-boson and q-fermion are very different. The monomials of quasi-Grassman variables cannot be obtained by naive replacement of $q$ by $-q$. Now using these results, the expression (9) can be written as

$$
\left(\psi \frac{d_{q}}{d_{q} \psi}\right)^{r} \phi(\psi)=\sum_{s=1}^{r} \mathcal{F}_{s}^{r}(\psi)^{s}\left(\frac{d_{q}}{d_{q} \psi}\right)^{s} \phi(\psi)
$$

and the expression (24) gives

$$
\left(\frac{d_{q}}{d_{q} \psi} \psi\right)^{r} \phi(\psi)=\sum_{s=1}^{r} \mathcal{B}_{s}^{r}\left(\frac{d_{q}}{d_{q} \psi}\right)^{s} \psi^{s} \phi(\psi) .
$$

Expressions (44) and (45) reveal the role of q-fermion Stirling number of the second kind and 'number $\mathcal{B}_{s}^{r}$ ' appearing in the anti-normal ordering of q-fermion operators, in expressing powers of q-differential operators.

\section{Summary}

We have considered the q-fermion numbers introduced in the q-fermion oscillator algebra by Parthasarathy and Viswanathan [6] in detail and obtained q-fermionic Stirling numbers of first and second kind explicitly. The 
q-fermionic Bell number is obtained by means of q-fermionic Dobinsky formula. q-fermionic Lah numbers are also considered. These results agree with those of Schork $[4,5]$. The case of 'anti-normal ordering' of q-fermionic annihilation and creation operators is studied and expansion coefficients $\mathcal{A}_{s}^{r}$ for q-bosonic operators and $\mathcal{F}_{s}^{r}$ for q-fermionic operators are introduced. Recurrence relations for these are derived and these are very different from those encountered in q-stirling numbers or q-Lah numbers. In this sense they are new. By taking matrix elements of the defining relations for these coefficients between q-bosonic and q-fermionic Fock space states, we obtain expressions for the powers of q-bosonic and q-fermionic numbers in terms of 'raising factorials'. These compliment the role of the q-Stirling numbers of the second kind as they express powers of q-bosonic and q-fermionic numbers in terms of 'falling factorials'. The theory of product densities of Ramakrishnan [10] is extended to q-stochastic point process and the necessity of introducing qproduct densities is emphasized. This leads to the identification of the effect of the degeneracy with q-fermionic Stirling numbers of the second kind. We have given a Bargmann space representation of q-fermion operators $F$ and $F^{\dagger}$ using q-fermion coherent states. This representation is used to express powers of q-differential operators acting on the space of entire functions of quasi-Grassmann variable as a series.

\section{References}

1. L.Carlitz, Trans.Amer.Math.Soc., 35 (1933) 122; H.W.Gould, Duke. Math.J., 28 (1961) 281; S.C.Milne, Trans.Amer.Math.Soc. 245 (1978) 89.

2. J.Katriel and M.Kibler, J.Phys.A:Math.Gen. 25 (1992) 2683.

3. A.J.Macfarlane, J.Phys.A:Math.Gen. 22 (1989) 4581.

L.C.Biedenharn, J.Phys.A:Math.Gen. 22 (1989) L873.

4. M.Schork, J.Phys.A:Math.Gen. 36 (2003) 4651.

5. M.Schork, J.Phys.A:Math.Gen. 36 (2003) 10391.

6. R.Parthasarathy and K.S.Viswanathan, J.Phys.A:Math.Gen. 24 (1991) 613. 
7. J.Katriel, Phys.Lett. A273 (2000) 159.

8. R.Parthasarathy and R.Sridhar, in Stochastic Point Processes, Editors: S.K.Srinivasan and A.Vijayakumar, Narosa Publishing House (New Delhi), 2003.

9. G.E.Andrews, The Theory of Partitions, (London: Addison-Wesley), 1976.

L.Comtet, Advanced Combinatorics, (Dordrecht: Reidel), 1974.

10. A.Ramakrishnan, Proc.Cambridge. Phil.Soc. 46 (1950) 595; ibid 48 (1952) 451; ibid 49 (1953) 473.

11. P.Narayana Swamy, Preprint, (1999), quant-ph/9909015.

12. M.Daoud, Y.Hassouni and M.Kibler, in: B.Gruber, M.Ramek (Eds). Symmetries in Science X, Plenum, New York, 1998; M.Daoud and M.Kibler, Phys.Lett. A321 (2004) 147.

13. K.S.Viswanathan, R.Parthasarathy and R.Jagannathan, J.Phys.A:Math.Gen. 24 (1992) L335.

14. C.G.Wagner, Preprint http://www.math.utk.edu/ wagner/papers/paper4.pdf

15. P.Blasiak, K.A.Penson and A.Solomon, Phys.Lett. A309 (2003) 198.

16. A.J.Bracken, D.S.MacAnally, R.B.Zhang and M.D.Gould, J.Phys.A:Math.Gen. 24 (1991) 1379.

17. R.Parthasarathy, Preprint IMSc.93/23, April 6, 1993. 